\title{
Hydrochemistry of Umm Er Radhuma Groundwater
}

\author{
Muhammad Gomaah ${ }^{1,2}$ \\ ${ }^{1}$ Desert Research Center, Cairo, Egypt \\ ${ }^{2}$ United Nations Developing Program (UNDP) \& Ministry of Env. Water and Agriculture, Riyadh, KSA \\ Email: muhammad_gomaah@yahoo.com
}

How to cite this paper: Gomaah, $M$ (2021). Hydrochemistry of Umm Er Radhuma Groundwater. Journal of Geoscience and Environment Protection, 9, 128-146. https://doi.org/10.4236/gep.2021.94008

Received: March 15, 2021

Accepted: April 22, 2021

Published: April 25, 2021

Copyright (c) 2021 by author(s) and Scientific Research Publishing Inc. This work is licensed under the Creative Commons Attribution International License (CC BY 4.0).

http://creativecommons.org/licenses/by/4.0/

\begin{abstract}
Umm Er Radhuma Aquifer (UER) is the most important groundwater aquifer in Saudi Arabia, extending over $1.6 \mathrm{M} \cdot \mathrm{km}^{2}$ in the eastern part of the Arabian Peninsula, forming the main aquifer throughout the Rub' Al-Khali (RAK). Groundwater salinity increased from west to east, reaching more than 27,000 $\mathrm{mg} / \mathrm{L}$ near the border with the United Arab Emirates, where a $\mathrm{Na}^{+}-\mathrm{Ca}^{2+} \mathrm{Cl}^{-}$$\mathrm{SO}_{4}^{2-}$ water type dominated. Gibbs diagrams indicated that the dissolution/precipitation of carbonates and evaporation/precipitation of minerals, especially anhydrite, gypsum, and halite account for the solutes and salinity in groundwater. Most of the samples plot above the 1:1 line of $\left(\mathrm{Ca}^{2+}+\mathrm{Mg}^{2+}\right)$ against $\mathrm{HCO}_{3}^{-}$, indicating other sources of $\mathrm{Ca}^{2+}$ and $\mathrm{Mg}^{2+}$ in the groundwater along with dolomite and calcium carbonate minerals. Phreeqc model indicated that the main clay minerals are kaolinite and gibbsite which had major effect on the cation exchange process as indicated by the Chloro-Alkaline index (CAI), where most of groundwater samples had values greater than zero which indicated the occurrence of reverse ion exchange between the groundwater and its host aquifer. The water type $\mathrm{Na}^{+}-\mathrm{Ca}^{2+} \mathrm{Cl}^{-}-\mathrm{SO}_{4}^{2-}$ dominated in the eastern part of the aquifer as the anaerobic conditions prevailed and the reduction of sulphate took place.
\end{abstract}

\section{Keywords}

Hydrochemistry, Umm Er Radhumma Aquifer, Groundwater, Water-Rock Interaction

\section{Introduction}

Groundwater chemistry is a function of many chemical and physical factors such as the mineral composition, lithology of the aquifer, oxidation reduction reac- 
tions, natural or artificial recharge and discharge, weathering, exchange reactions (Elango \& Kannan, 2007). Understanding the hydrochemistry of groundwater is of great importance, especially in such arid areas like Saudi Arabia where groundwater provides the major water supply.

The Kingdom of Saudi Arabia is commonly regarded as a dry country due to low precipitation and high evaporation rate. The mean annual rainfall across the Arabian Peninsula is less than $200 \mathrm{~mm}$, and the temperature reaches more than $50^{\circ} \mathrm{C}$ in summers. Though no perennial surface water in the kingdom, groundwater is the most important natural water resource which provides the kingdom with water supply for domestic and agricultural purposes. Among the most important groundwater reservoirs in Saudi Arabia is Umm Er Radhuma aquifer (UER) as many of Arab Gulf states depend on it for different purposes. It is extending over $1.6 \mathrm{M} \cdot \mathrm{km}^{2}$ in the eastern part of the Arabian Peninsula, forming the main aquifer throughout the Rub' al Khali (RAK) Desert where it is located in the southern areas of Saudi Arabia (Figure 1). RAK is considered the world's largest continuous sand desert, covering an area of 522,340 $\mathrm{km}^{2}$.

The Umm Er Radhuma (UER) aquifer has been investigated geologically, hydrogeologically since the early 1940s. Some of the previous research is summarized by Powers et al. (1966), Sogreah (1968), Watuki (1968, 1971), Iltaloconsult (1969), Dinçer et al. (1974), BRGM (1976, 1979), GDC (1979), Shampine et al. (1979), Al Bassam (1983, 1987), MOWE (2008). MEWA (2017) studied the general hydrochemistry of Umm Er Radhuma aquifer. However, further hydrochemistry investigations are required to identify in detail the origin and sources of salinity in the groundwater. This is what the current paper is trying to seek.

\section{Geology and Hydrogeology}

The Late Paleocene to Early Eocene sediments of Umm Er Radhuma formation

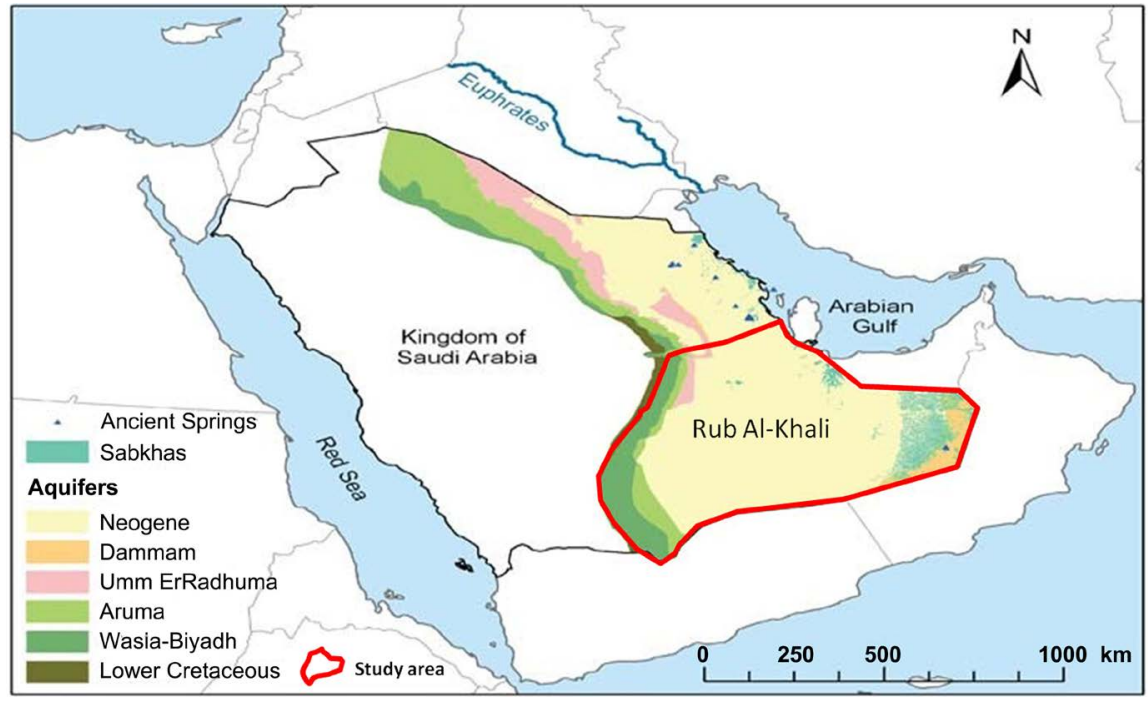

Figure 1. Location of the study area showing the extent and outcrop of the Umm Er Radhuma aquifer (after Edda et al., 2011). 
had deposited during major transgression where marine conditions prevailed on the Arabian Peninsula as far as Jordan, Iraq, Oman and Yemen (Ziegler, 2001). Calcareous shales deposited at the base of the formation followed by thick succession of carbonate rocks. The sedimentation took place on a wide carbonate shelf, which was distorted partly by tectonic movements during the movement of the Arabian plate into a series of deeps and shallows (Figure 2). A compressional force during the movement led the hypersaline groundwater from the Wasia-Biyadh aquifer to be injected through the deep faults into the overlying beds. This process might interpret the dolomitization of Umm Er Radhuma limestone, in which the dolomite replaced calcite and aragonite in association with the precipitation of secondary anhydrite. On the other hand, the dolomitization in the southern portion might be attributed to the leakage from the overlying anhydrite and evaporates bed (Rus formation). Thick calcarenite beds and reef type carbonates are contained in the central and southern part of the formation which were deposited around paleo-highs. Marls and shales occur between the carbonate units of the upper part of the formation, while cherts occur sporadically throughout the central and southern part. The formation crops out along the northwest-southeast trending belt from Rafha in the north to east of $\mathrm{Al}$ Kharj with a mean thickness of about $350 \mathrm{~m}$, and it gets thicker in the central part of the Rub' Al Khali Basin, reaching more than 600 m (MEWA, 2017). The transmissivity ranges between $7.2 \times 10^{-5} \mathrm{~m}^{2} / \mathrm{s}$ from $5.2 \times 10^{-1} \mathrm{~m}^{2} / \mathrm{s}$, and the conductivity varies between $2.9 \times 10^{-7} \mathrm{~m} / \mathrm{s}$ and $8.8 \times 10^{-3} \mathrm{~m} / \mathrm{s}$, with an average of 1.6 $\times 10^{-5} \mathrm{~m} / \mathrm{s}$. The wide range of the values is caused by the varying degree of karstification. The storage coefficient varies between $3 \times 10^{-4}$ and $3 \times 10^{-3}$ while the specific yield ranges from $S y=1 \%$ to $7 \%$ (MEWA, 2017).

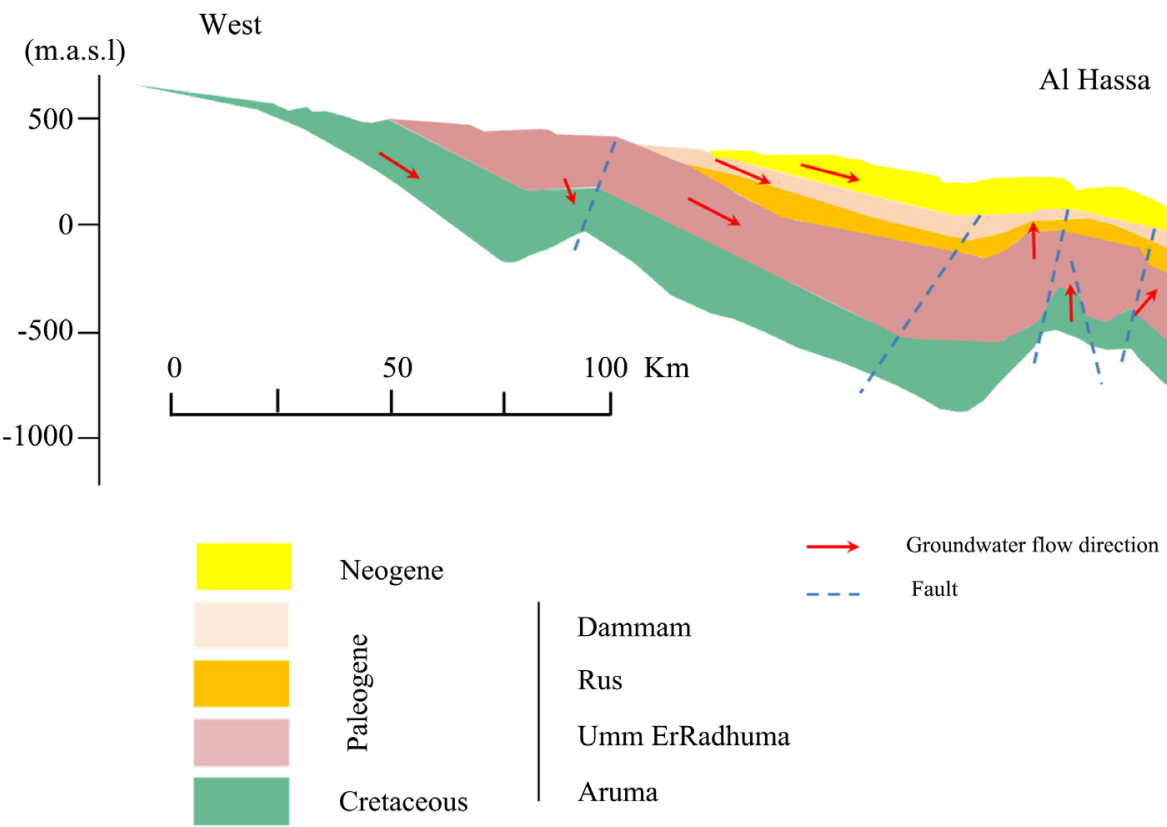

Figure 2. Schematic diagram illustrating the dolomitization of the limestone through interaction withhigh saline groundwater from the underlying aquifers. 


\section{Materials and Methods}

\section{Groundwater Sampling and Laboratory Analyses}

The data in this paper was carried out by the Ministry of Environment Water and Agriculture in association with the Deutsche Gesellschaft für Internationale Zusammenarbeit (GIZ) as an internal report project, titled "Detailed Groundwater Resources Studies in The Rub' Al Khali Desert”, in addition to a historic data compiled from BRGM (1976). 44 groundwater samples have been collected by the GIZ during field trips from November 2010 to December 2011. Three samples were taken at each well for TDS, major cations, and major anions. Forty four Groundwater samples have been collected in $100 \mathrm{ml}$ polyethylene screw-cap bottles which were rinsed three times with the water to be sampled. All samples were filtered using $0.45 \mu \mathrm{m}$ filter and preserved by cooling. Additionally, nitric acid $\left(\mathrm{HNO}_{3} 70 \%\right)$ has been added to samples dedicated for cation analysis for further preservation. Field measurements included pH, EC, T, dissolved oxygen, bicarbonate and hydrogen sulfide using a flow-through-cell and multi-parameter pocket meters (Multi 340i, WTW, Germany). Moreover, groundwater samples were collected for trace elements in $250 \mathrm{ml}$ polyethylene bottles. Samples were then shipped in cooling boxes to the hydrochemical laboratory in Darmstadt Technical University, Germany. The following standard analytical techniques were applied to investigate the hydrochemical composition of groundwater samples:

- Ion chromatography (IC; major ions);

- Atomic Adsorption Spectrometry (AAS; $\mathrm{Fe}^{2+}$ );

- Photometric tests $\left(\mathrm{B}, \mathrm{SiO}_{2}\right)$;

- Gravimetry (TDS);

- Atomic Adsorption Spectrometry, ICP/MS, and ICP-OES (trace elements).

Phreeqc is a computer program written in the $\mathrm{C}$ programming language which has been designed to conduct a broad variety of aqueous geochemical calculations. Phreeqc has capabilities for 1) calculation of speciation and saturation-index, 2) reaction-path and advective-transport calculations involving specified irreversible reactions, mixing of solutions, mineral and gas equilibria, surface-complexation reactions, and ion-exchange reactions, and 3) inverse modeling, which identifies the mole transfers that account for composition changes between waters a long flow path (Parkhurst, 1995).

\section{Results and Discussion}

According to the statistical summary of the hydrochemical data of the groundwater samples of Umm Er Radhuma aquifer (Table 1 \& Figure 3), most of the samples are fresh $(<1000 \mathrm{mg} / \mathrm{l})$ to slightly saline near the outcrops (Figure 3 ), where it is characterized by a $\mathrm{Ca}^{2+}-\mathrm{SO}_{4}^{2-}$ water type. Low salinity is also observed in the Dhofar Mountains (Oman) due to freshwater recharge that may be responsible for the lower saline groundwater tongue extending from the Dhofar Mountains to the north. However, salinity increases from west to east, where the 


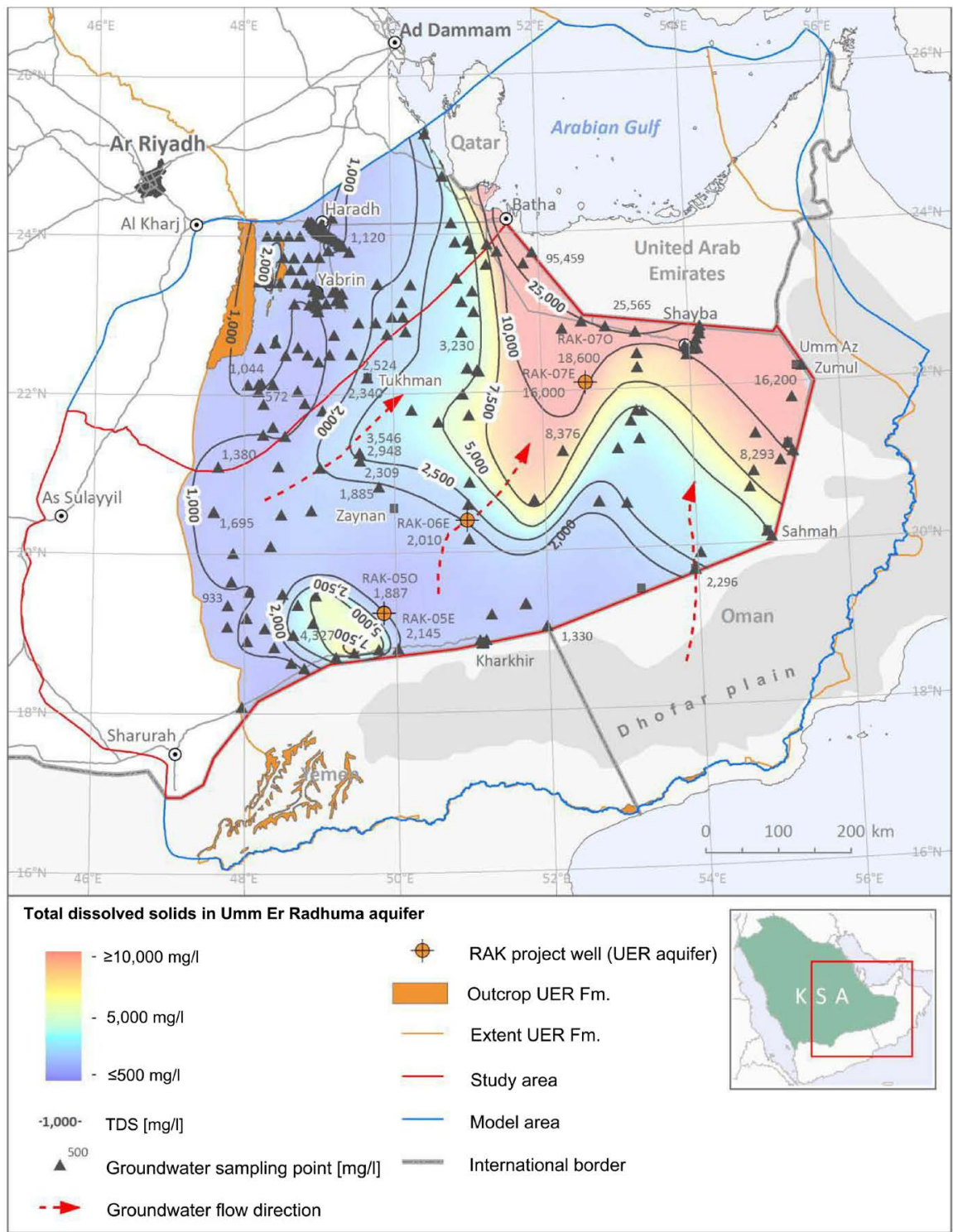

Figure 3. TDS distribution of the groundwater samples for Umm Er Radhuma aquifer.

Table 1. Chemical analyses data for the groundwater of Umm Er Radhuma aquifer (in ppm).

\begin{tabular}{ccccccccccccc}
\hline Well & LAT & LONG & $\mathrm{pH}$ & $\mathrm{EC} \mu \mathrm{S} / \mathrm{cm}$ & $\mathrm{TDS}$ & $\mathrm{Ca}^{2+}$ & $\mathbf{M g}^{2+}$ & $\mathrm{Na}^{+}$ & $\mathrm{K}^{+}$ & $\mathrm{Cl}^{-}$ & $\mathbf{H C O}_{3}^{-}$ & $\mathbf{S O}_{4}^{2-}$ \\
\hline GHFH-801 & 21.02850 & 55.16011 & 7.19 & 15,910 & 10,900 & 477.0 & 271.0 & 2570 & 76.1 & 5180 & 216.6 & 972.0 \\
OLTD40 & 22.39989 & 53.96625 & 7.90 & 16,500 & 10,800 & 501.0 & 186.0 & 2920 & 93.9 & 5300 & 308.1 & 697.0 \\
S-397 & 23.44653 & 50.89114 & 7.38 & 591.0 & 4810 & 591.0 & 250.0 & 998.0 & 53.9 & 734.0 & 115.9 & 2330 \\
OLTD13 & 23.86678 & 51.30725 & 8.79 & 9670 & 6870 & 485.0 & 199.0 & 1340 & 72.6 & 2360 & 137.3 & 1830 \\
OLTD52 & 22.19956 & 55.50636 & 7.00 & 33,800 & 4100 & 1050 & 395.0 & 5130 & 113.0 & 8150 & 176.1 & 2210 \\
OLTD39 & 22.46494 & 53.98664 & 6.80 & 17,720 & 10,700 & 569.0 & 193.0 & 3060 & 98.2 & 5690 & 332.5 & 651.0 \\
JZTD2 & 18.76103 & 49.44333 & 7.34 & 12,320 & 7285 & 630.0 & 362.0 & 1660 & 95.1 & 3270 & 48.8 & 1940 \\
MKSR-801 & 21.26433 & 49.54578 & 6.72 & 2610 & 2309 & 254.0 & 99.5 & 213.0 & 34.5 & 142.0 & 277.6 & 1160 \\
7-S-11 & 20.53239 & 47.59878 & 6.79 & 2740 & 1744 & 210.7 & 78.6 & 224.3 & 10.0 & 594.8 & 173.9 & 395.3 \\
\hline
\end{tabular}


Continued

\begin{tabular}{|c|c|c|c|c|c|c|c|c|c|c|c|c|}
\hline $7-S-25$ & 22.71242 & 53.28344 & 7.75 & 66,000 & 49,500 & 2520 & 896.0 & 13,300 & 351.0 & 32,200 & 491.2 & 1230 \\
\hline SUHL-801 & 21.59570 & 53.18430 & 7.15 & 5390 & 3739 & 201.1 & 102.5 & 814.0 & 53.6 & 1.585 & 213.5 & 1.091 \\
\hline KharkherNewWell & 18.90000 & 51.18339 & 7.68 & 2270 & 1932 & 194.0 & 105.0 & 118.0 & 15.0 & 176.0 & 57.5 & 848.0 \\
\hline JZTD1 & 18.08494 & 47.96872 & 7.09 & 2730 & 1770 & 101.0 & 52.2 & 364.0 & 9.1 & 605.0 & 50.0 & 368.0 \\
\hline KIDNSRAK-804 & 21.71410 & 53.34540 & 7.47 & 7530 & 5782 & 453.0 & 176.0 & 1110 & 77.1 & 1410 & 216.6 & 1800 \\
\hline RAK-05O & 19.25069 & 49.84586 & 6.96 & 2480 & 1887 & 242.3 & 98.1 & 194.9 & 12.8 & 253.7 & 73.0 & 928.9 \\
\hline FYDH-LKSR-801 & 22.14967 & 48.23289 & 6.90 & 1650 & 1044 & 118.9 & 47.1 & 161.7 & 25.3 & 275.6 & 234.9 & 277.6 \\
\hline RAK-08O & 20.83889 & 49.79500 & 6.97 & 2660 & 1885 & 303.4 & 106.9 & 181.6 & 23.1 & 183.7 & 186.1 & 1069 \\
\hline RAK-08O & 20.83889 & 49.79500 & 6.97 & 2660 & 1885 & 303.4 & 106.9 & 235.2 & 17.9 & 303.1 & 183.0 & 889.5 \\
\hline RAK-06 & 20.39725 & 50.96567 & 7.05 & 2730 & 2010 & 236.2 & 111.2 & 74.7 & 15.0 & 133.6 & 228.2 & 137.8 \\
\hline FAYD-801 & 22.04542 & 48.19744 & 7.40 & 1000 & 572 & 51.4 & 44.3 & 190.6 & 28.9 & 174.9 & 186.1 & 1262 \\
\hline TKMN-803 & 22.20667 & 49.65689 & 7.45 & 2590 & 2340 & 278.9 & 119.4 & 219.7 & 36.3 & 125.6 & 256.2 & 1.28 \\
\hline RAK-EX-04 & 21.26444 & 49.54583 & 6.77 & 2680 & 1948 & 294.4 & 113.2 & 188.3 & 28.7 & 176.2 & 183 & 1.255 \\
\hline RAK-EX-07 & 22.20761 & 49.66111 & 7.40 & 2860 & 2524 & 271.5 & 120.6 & 177.2 & 43.0 & 101 & 152.5 & 2.303 \\
\hline RAK-EX-05A & 21.16306 & 49.54611 & 7.10 & 3200 & 3546 & 505.0 & 192.2 & 177.0 & 8.3 & 316 & 50 & 254 \\
\hline HFER-RBKL-801 & 23.09711 & 48.98550 & 6.87 & 1749 & 1140 & 105.0 & 58.5 & 94.1 & 7.0 & 178.0 & 149.5 & 344.0 \\
\hline Well Al Bragh & 21.79833 & 49.05378 & 7.27 & 1399 & 1000 & 104.0 & 55.9 & 206.0 & 22.0 & 258.0 & 170.8 & 1140 \\
\hline HDDH-801 & 21.79567 & 50.24681 & 7.05 & 2760 & 2320 & 257.0 & 107.0 & 527.0 & 47.1 & 774.0 & 161.7 & 1520 \\
\hline S-610 & 23.28025 & 51.09647 & 7.28 & 4960 & 3690 & 332.0 & 162.0 & 618.0 & 45.9 & 999.0 & 170.8 & 1300 \\
\hline$S-646$ & 22.31478 & 51.00728 & 7.26 & 5310 & 3780 & 286.0 & 151.0 & 667.0 & 48.3 & 989.0 & 167.8 & 1220 \\
\hline S-612 & 23.02275 & 51.10889 & 7.30 & 5220 & 3650 & 268.0 & 148.0 & 1180 & 60.4 & 1970 & 192.2 & 1100 \\
\hline Grada-01 & 22.27097 & 51.15928 & 7.34 & 8500 & 5200 & 257.0 & 140.0 & 135.0 & 5.9 & 1750 & 198.3 & 1230 \\
\hline Grada-WW1 & 22.28719 & 51.14258 & 7.36 & 7970 & 4990 & 284.0 & 154.0 & 167.0 & 12.8 & 238 & 110.5 & 337 \\
\hline $7-S-64$ & 18.57017 & 48.78839 & 7.07 & 1777 & 1220 & 124.0 & 48.2 & 442.0 & 42.7 & 44.9 & 149.5 & 977 \\
\hline Sul_12 & 20.07320 & 54.99260 & 7.01 & 8880 & 5358 & 386.0 & 110.0 & 499.0 & 36.1 & 629 & 146.4 & 882 \\
\hline $7-S-29$ & 19.71080 & 53.96380 & 7.34 & 3520 & 2575 & 183.0 & 122.0 & 148.9 & 9.1 & 651.2 & 158.6 & 718.6 \\
\hline W7-S-84 & 19.91240 & 54.04070 & 7.27 & 3730 & 2573 & 142.9 & 106.3 & 335.6 & 23.5 & 237.9 & 222.7 & 974.5 \\
\hline RAK-05E & 19.25117 & 49.84561 & 6.95 & 2430 & 2145 & 300.8 & 106.8 & 135.0 & 17.3 & 498.5 & 161.7 & 1128 \\
\hline FRIS-801 & 20.40790 & 50.94010 & 7.00 & 2930 & 2260 & 189.2 & 88.8 & 138.1 & 19.1 & 199.0 & 161.7 & 872.0 \\
\hline 7-S-XX & 18.85581 & 51.12736 & 7.03 & 2200 & 1730 & 206.0 & 110.0 & 152.0 & 19.2 & 219.4 & 164.7 & 1079 \\
\hline KharkherBGCenter & 18.89780 & 51.10250 & 6.99 & 2300 & 1905 & 215.4 & 116.0 & 886.8 & 38.7 & 236.0 & 58.5 & 1010 \\
\hline Kharkher_Desal & 18.85967 & 51.13067 & 6.97 & 2230 & 1821 & 231.0 & 120.0 & 306.0 & 18.5 & 1.504 & 186.1 & 831.8 \\
\hline $7-S-28$ & 20.13660 & 54.93510 & 8.21 & 5550 & 3710 & 121.3 & 110.7 & 200.7 & 12.2 & 411.0 & 243.8 & 522.0 \\
\hline ST16 & 19.34767 & 51.71422 & 6.90 & 2460 & 1530 & 143.0 & 72.4 & 159.0 & 23.2 & 291.7 & 113.8 & 1147 \\
\hline UumAlMulahWell & 18.80419 & 50.02075 & 6.92 & 2810 & 2399 & 302.5 & 123.9 & 519.0 & 29.5 & 252.0 & 45.5 & 750.0 \\
\hline $7-S-53$ & 19.21733 & 51.25808 & 7.00 & 2200 & 1749 & 175.0 & 92.2 & 420.0 & 28.8 & 841.0 & 210.5 & 874.0 \\
\hline S-699A & 20.56910 & 53.09450 & 7.07 & 4010 & 2825 & 233.0 & 97.7 & 159.8 & 12.8 & 712.0 & 204.4 & 533.0 \\
\hline S-688A & 20.60100 & 52.72340 & 7.17 & 3260 & 2717 & 3260 & 7.17 & 757.0 & 38.5 & 238.0 & 205.1 & 547.2 \\
\hline
\end{tabular}




\begin{tabular}{|c|c|c|c|c|c|c|c|c|c|c|c|c|}
\hline KRKR-803 & 19.05739 & 51.98522 & 7.00 & 1966 & 1482 & 129.6 & 97.3 & 131.6 & 16.9 & 1140 & 207.4 & 646.0 \\
\hline SRFH-801 & 21.37140 & 53.28830 & 7.32 & 4660 & 3043 & 143.0 & 91.0 & 2410 & 144.0 & 203.8 & 113.5 & 941.6 \\
\hline JZTD7 & 18.86703 & 51.14075 & 7.01 & 2290 & 1945 & 232.0 & 118.3 & 4000 & 98.2 & 203.8 & 113.5 & 941.6 \\
\hline S-686 & 20.64190 & 51.85880 & 6.92 & 13,810 & 10,329 & 465.0 & 339.0 & 2810 & 77.8 & 3850 & 216.6 & 2230 \\
\hline RWKB-801 & 21.80960 & 55.35010 & 7.18 & 22,600 & 16,200 & 559.0 & 260.0 & 6130 & 231.0 & 7760 & 271.5 & 701.0 \\
\hline $7-S-27$ & 21.23760 & 55.26480 & 7.29 & 16,500 & 10,589 & 407.0 & 219.0 & 4580 & 151.0 & 5110 & 216.6 & 1020 \\
\hline RAK-07O & 22.09081 & 52.59347 & 6.94 & 27,100 & 18,600 & 1010 & 381.0 & 440.0 & 17.4 & 9250 & 384.3 & 930.0 \\
\hline RA K-07E & 22.09064 & 52.59383 & 6.76 & 25,000 & 16,000 & 726.0 & 254.0 & 549.0 & 26.4 & 9580 & 100.0 & 789.0 \\
\hline
\end{tabular}

total dissolved solids (TDS) value reached more than 27,000 mg/L near the border with the United Arab Emirates, where a $\mathrm{Na}^{+}-\mathrm{Ca}^{2+} \mathrm{Cl}^{-}-\mathrm{SO}_{4}^{2-}$ water type dominates (Figure 4).

\section{Water-Rock Interaction and Sources of Solutes}

Gibbs diagrams are considered an effective tool to identify the main geochemical processes which control the hydrogeochemical characteristics of groundwater in aquifers such as atmospheric precipitation, rock weathering, evaporation, and minerals precipitation. The diagrams describe the weight ratios of $\mathrm{Na}^{+} /\left(\mathrm{Na}^{+}+\right.$ $\left.\mathrm{Ca}^{2+}\right)$ and $\mathrm{Cl}^{-} /\left(\mathrm{Cl}^{-}+\mathrm{HCO}_{3}^{-}\right)$against TDS as shown in Figure 5, where most of the samples plot between the rock weathering and the evaporation zones. Therefore, the dissolution/precipitation of carbonates and evaporation/precipitation of minerals, especially anhydrite, gypsum, and halite are the major geochemical reactions which account for the solutes in groundwater.

The dissolution of carbonate minerals takes place when carbon dioxide infiltrates along with rainfall and surface water into the aquifer near the outcrops, leading to the formation of carbonic acid which dissociates carbonate minerals in the investigated aquifer according to the following equations:

$$
\begin{gathered}
\mathrm{H}_{2} \mathrm{O}+\mathrm{CO}_{2}=\mathrm{H}_{2} \mathrm{CO}_{3}=\mathrm{H}^{+}+\mathrm{HCO}_{3} \\
\mathrm{CaCO}_{3}+\mathrm{H}^{+} \rightarrow \mathrm{Ca}^{2+}+\mathrm{HCO}_{3} \\
\mathrm{CaMg}\left(\mathrm{CO}_{3}\right)_{2}+2 \mathrm{H}_{2} \mathrm{O}+2 \mathrm{CO}_{2} \rightarrow \mathrm{Ca}^{2+}+\mathrm{Mg}^{2+}+4 \mathrm{HCO}_{3}^{-} \\
\mathrm{CaSO}_{4} \rightarrow \mathrm{Ca}^{2+}+\mathrm{SO}_{4}^{2-}
\end{gathered}
$$

The dissolution of dolomite is well illustrated in Figure 6, where most of the cores of the dolomite rhombs undergo dissolution, which release equally charged amounts of $\left(\mathrm{Ca}^{2+}+\mathrm{Mg}^{2+}\right)$ and $\mathrm{HCO}_{3}^{-}$, respectively, according to Equations (2) and (3). Accordingly, samples should plot along the 1:1 line if dolomite is the only source of $\mathrm{Ca}^{2+}$ and $\mathrm{Mg}^{2+}$. According to Figure 7, most of the samples plot above the 1:1 line of $\left(\mathrm{Ca}^{2+}+\mathrm{Mg}^{2+}\right)$ against $\mathrm{HCO}_{3}^{-}$, indicating other sources of $\mathrm{Ca}^{2+}$ and $\mathrm{Mg}^{2+}$ in the groundwater along with dolomite and Calcium carbonate minerals. 


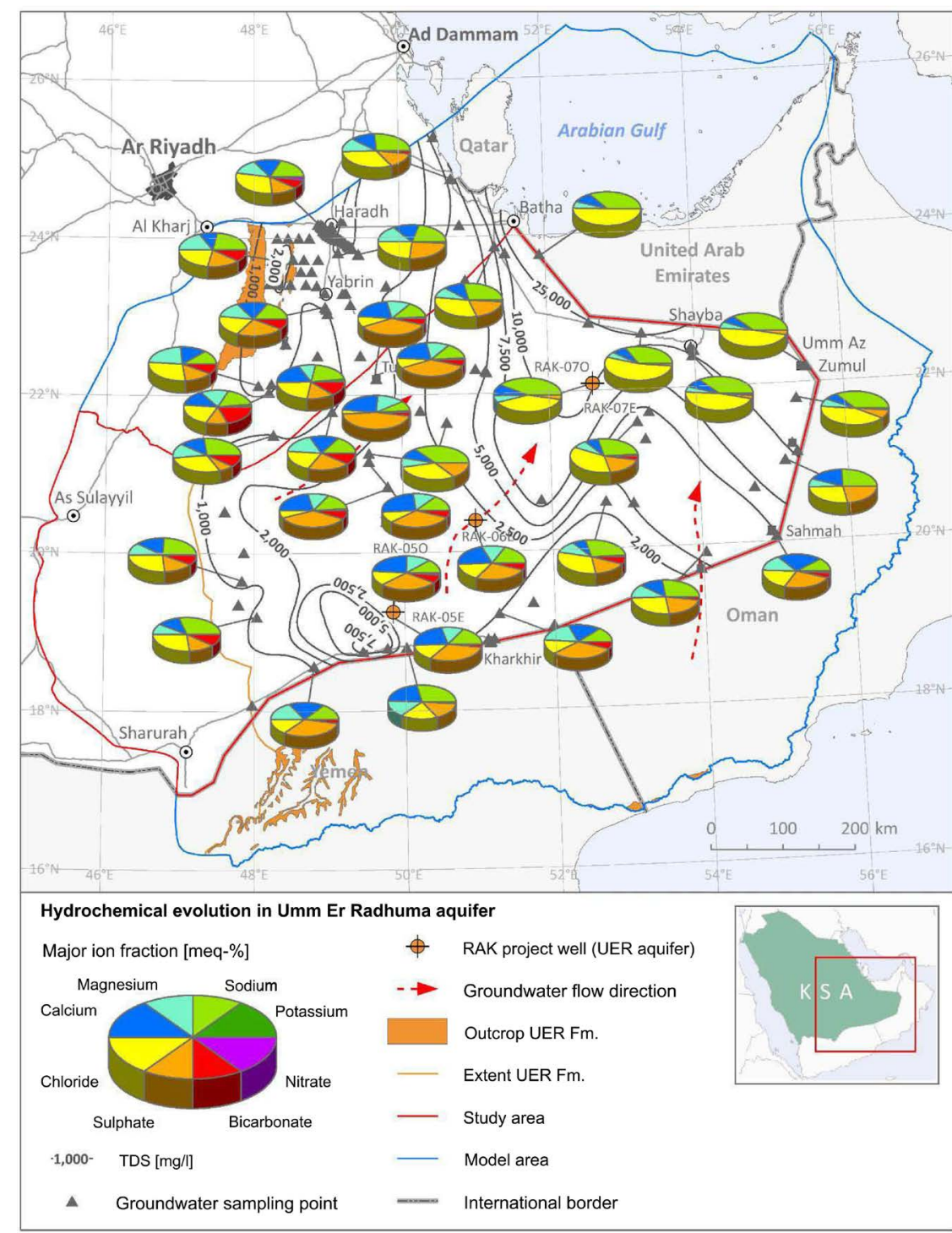

Figure 4. Water type distribution for groundwater samples of Umm Er Radhuma aquifer.

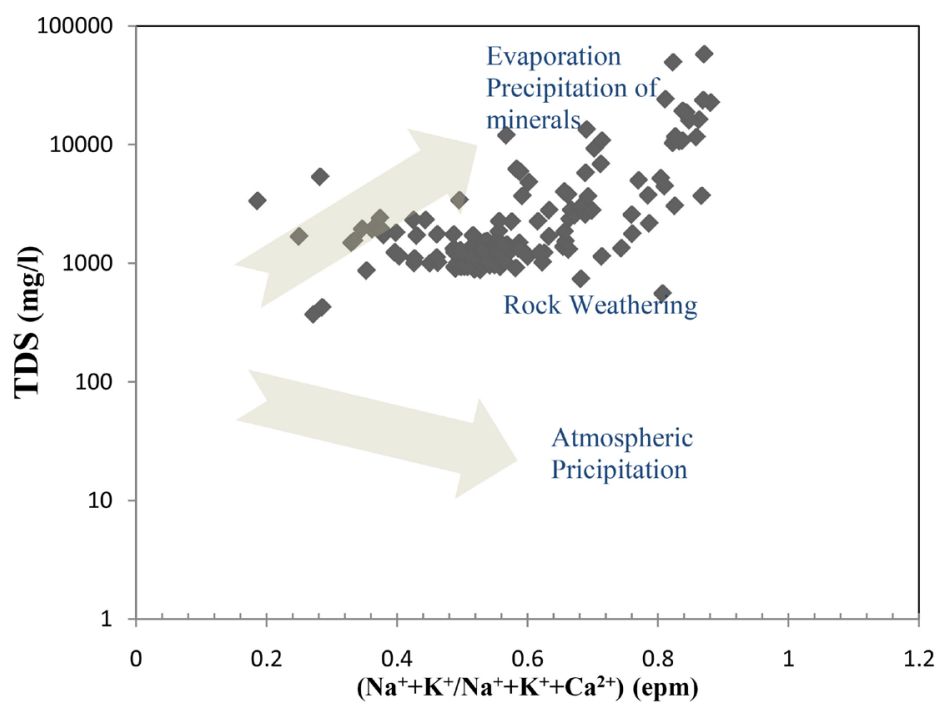




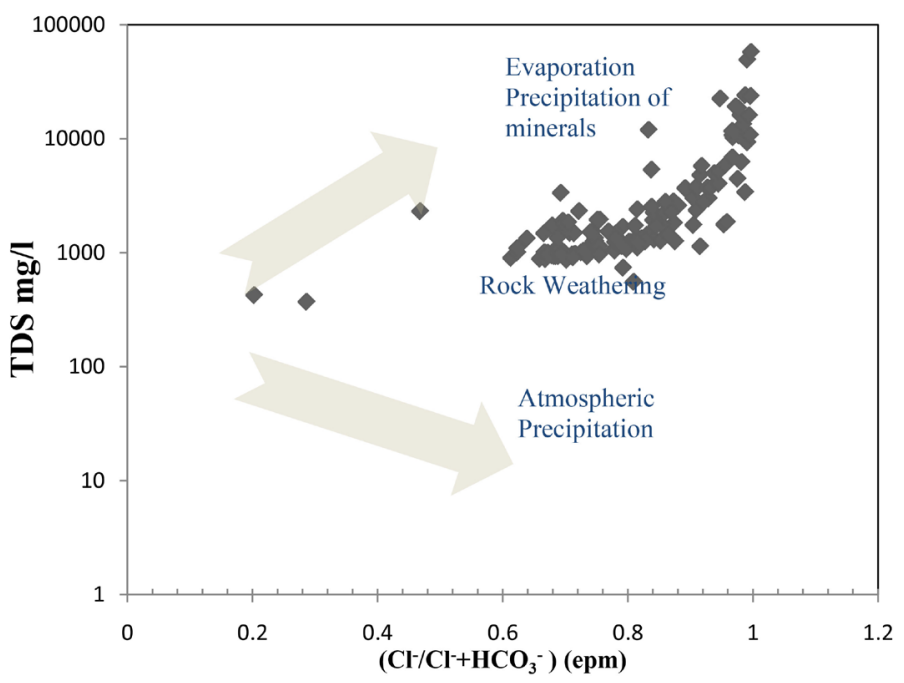

Figure 5. Gibbs diagrams, illustrating the main geochemical mechanisms in groundwater.

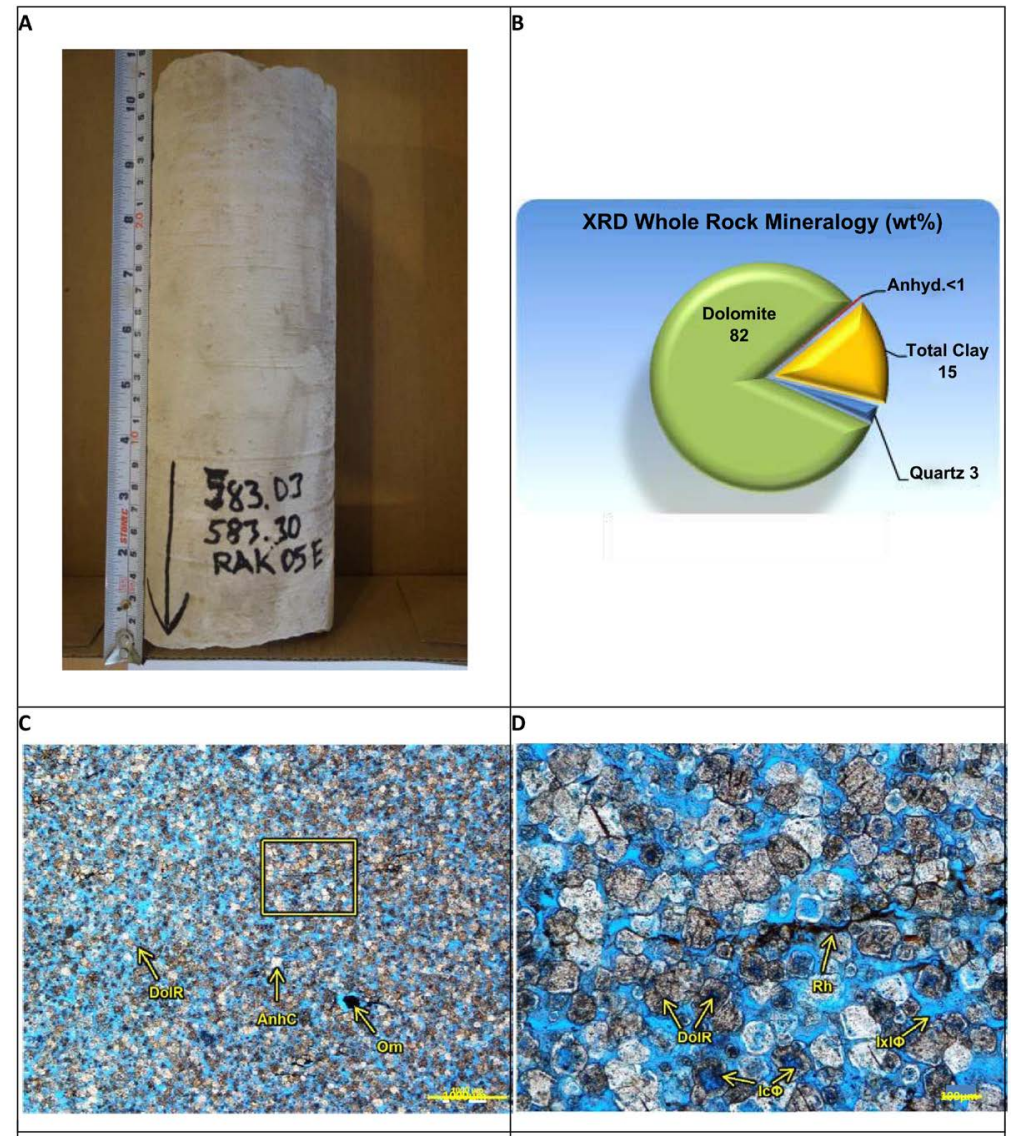

Figure 6. Laboratory results RAK 05-E. (A) Core sample RAK 05-E. (B) Pie chart showing the results from the X-Ray Diffraction (XRD) Analysis. Core sample RAK 05-E. (C) Low magnification view showing a dolostone sample with abundant replacive dolomite rhomb (DolR), minor anhydrite cement (AnhC) and trace opaque organic matter (Om). (D) High magnification view highlighting abundant fine to medium crystalline, cloudy to clear dolomite rhomb (DolR) with intercrystalline pores (Ixl $\Phi)$ and intracrystalline pores reflecting leaching of dolomite cores (Ic $\Phi)$. Minor residual hydrocarbon (Rh) within intercrystalline space observed (MEWA, 2017). 


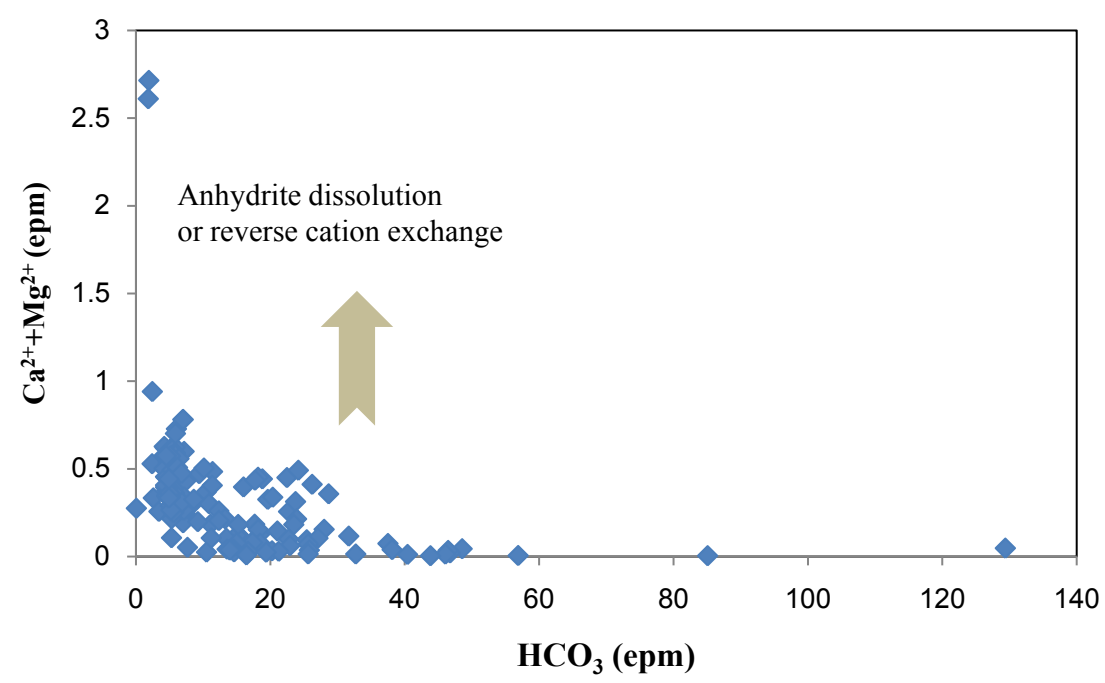

Figure 7. $\mathrm{Ca}^{2+}+\mathrm{Mg}^{2+}$ against $\mathrm{HCO}_{3}^{2-}$ relationship, indicating dolomite dissolution as a source of $\mathrm{Ca}^{2+}$ and $\mathrm{Mg}^{2+}$ along with anhydrite dissolution and reverse cation exchange.

In order to investigate the other sources of $\mathrm{Ca}^{2+}$ and $\mathrm{Mg}^{2+}$, samples were plot on $\mathrm{Ca}^{2+}$ against $\mathrm{SO}_{4}^{2-}$ relationship, illustrating the dissolution of anhydrite which would contribute more $\mathrm{Ca}^{2+}$ to groundwater if the samples plot along the 1:1 line for the anhydrite dissolution. Based on Figure 8, most of the samples plot along the 1:1 line except for a few samples which plot above and below the 1:1 line. The excess of $\mathrm{Ca}^{2+}$ above the 1:1 line suggests another source of $\mathrm{Ca}^{2+}$ along with anhydrite dissolution which might be cation exchange, whilst the decrease in $\mathrm{Ca}^{2+}$ is probably due to precipitation of calcite and dolomite for some groundwater samples. This has been emphasized by the examination of the saturation indices of carbonate minerals (Figure 9 \& Figure 10) which show that nearly most of the samples located in the eastern part of the area are saturated to supersaturated with respect to calcite and dolomite, except for a few samples located in or close to the outcrops where the recharge occurs and the oxic condition prevails. On the other hand, almost all samples are strongly undersaturated with respect to gypsum and anhydrite (Figure 11, Figure $12 \& \mathrm{Ta}$ ble 2).

Due to the abundancy of the organic matter in the middle and eastern parts along with the depletion of oxygen downgradient, sulfate reducing bacteria (SRB) become more active where sulfate are used as an electron acceptor while degrading the organic matter. Accordingly, sulfate becomes very depleted in the groundwater by reduction into hydrogen sulphide (Equation (5)). Hence, the water type $\mathrm{Na}^{+}-\mathrm{Ca}^{2+} \mathrm{Cl}^{-}-\mathrm{SO}_{4}^{2-}$ dominates in this part of the aquifer. In addition, the released acids and carbon dioxide by the degradation of the organic matter facilitate the dissolution of calcite (Figure 13).

$$
\text { Organic matter }+\mathrm{SO}_{4}^{2-}=\mathrm{H}_{2} \mathrm{~S}
$$

According to the result of the saturation indices conducted by phreeqc for the well RAK 05-E (Table 3), the main clay minerals are kaolinite and gibbsite 


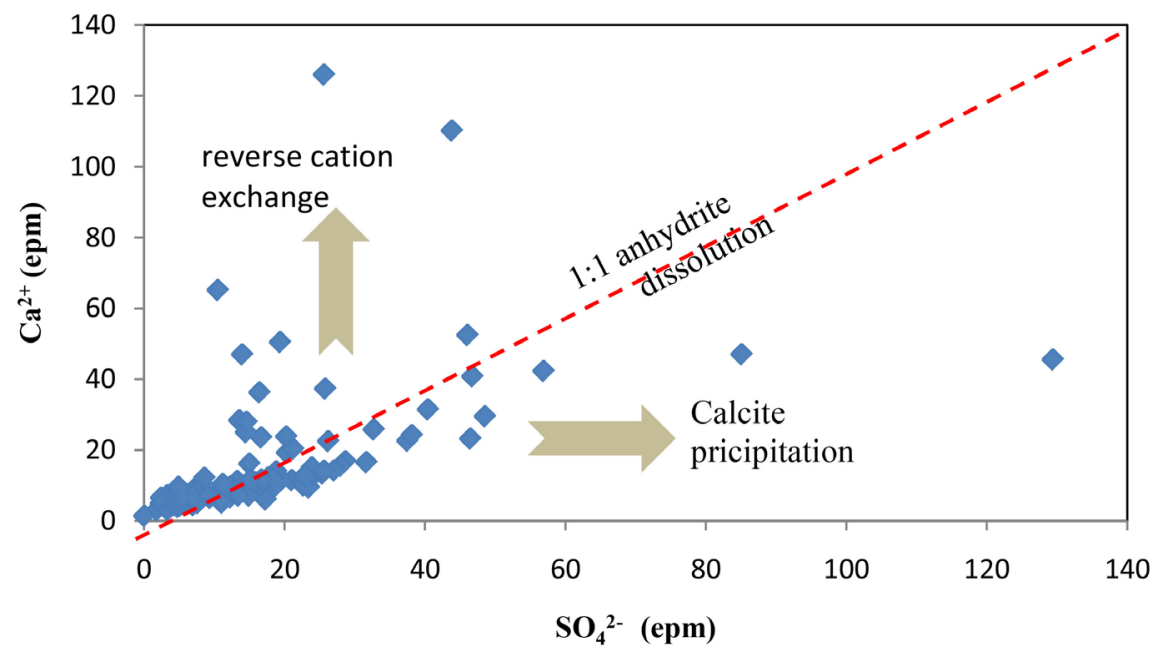

Figure 8. $\mathrm{Ca}^{2}$ against $\mathrm{SO}_{4}^{2-}$ relationship, indicating the dissolution of anhydrite when samples plot along the 1:1 line.

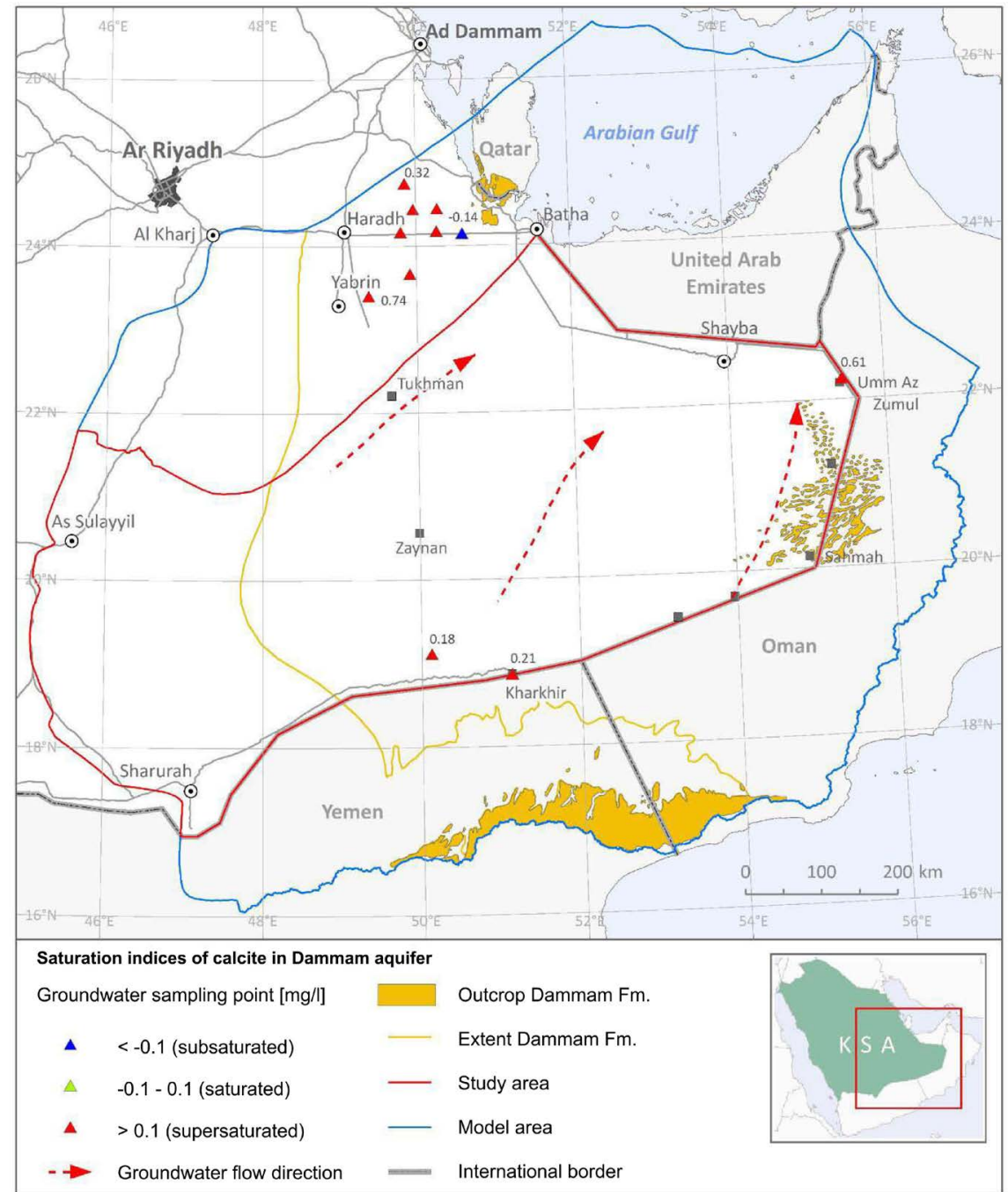

Figure 9. Distribution of the saturation indices of calcite within Umm Er Radhuma aquifer. 


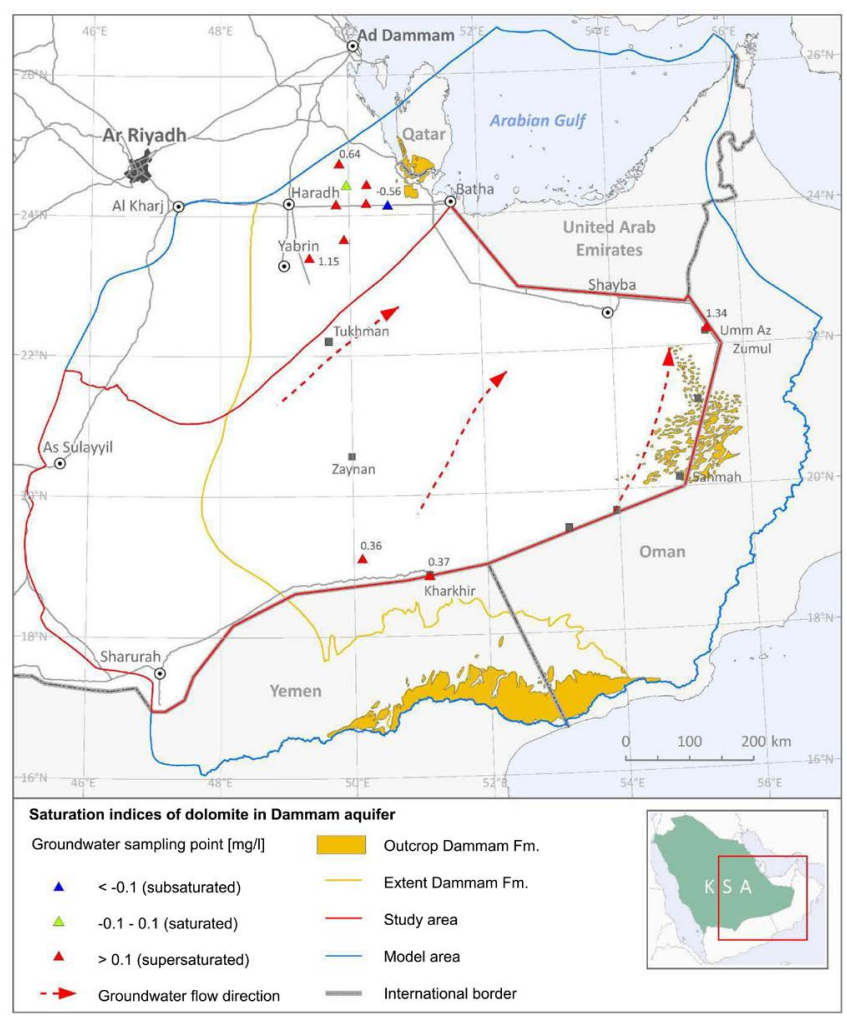

Figure 10. Distribution of the saturation indices of dolomite within Umm Er Radhuma aquifer.

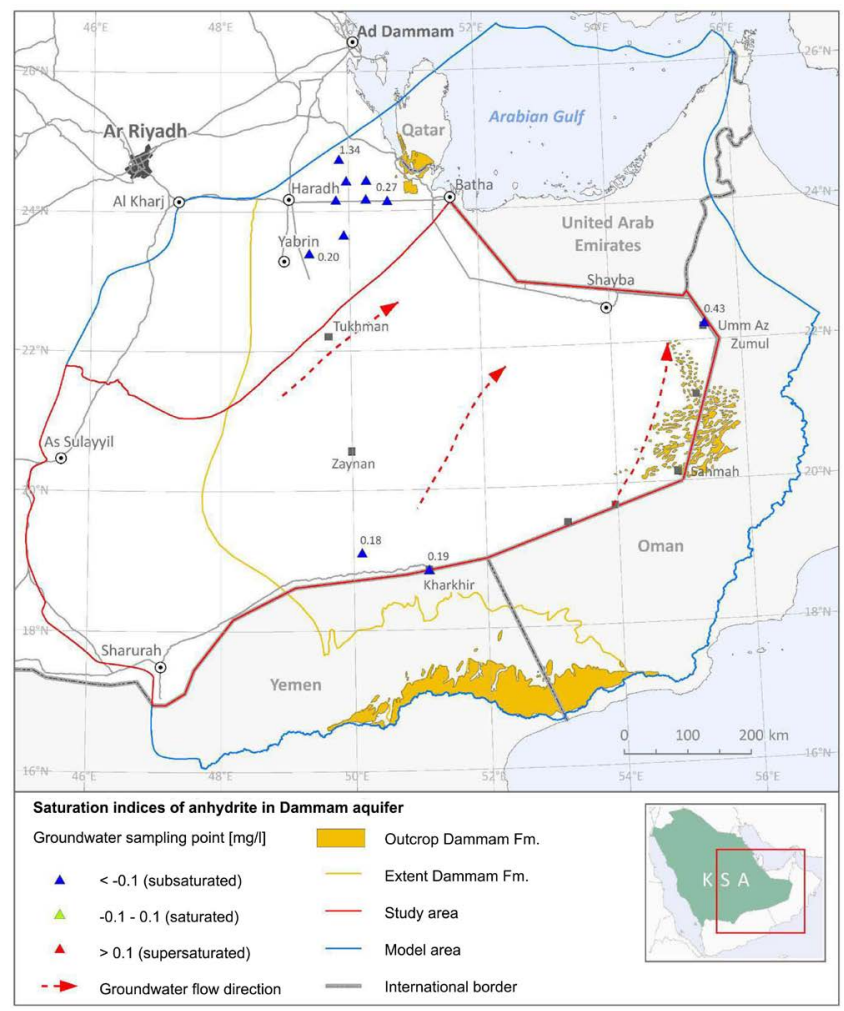

Figure 11. Distribution of the saturation indices of anhydrite within Umm Er Radhuma aquifer. 


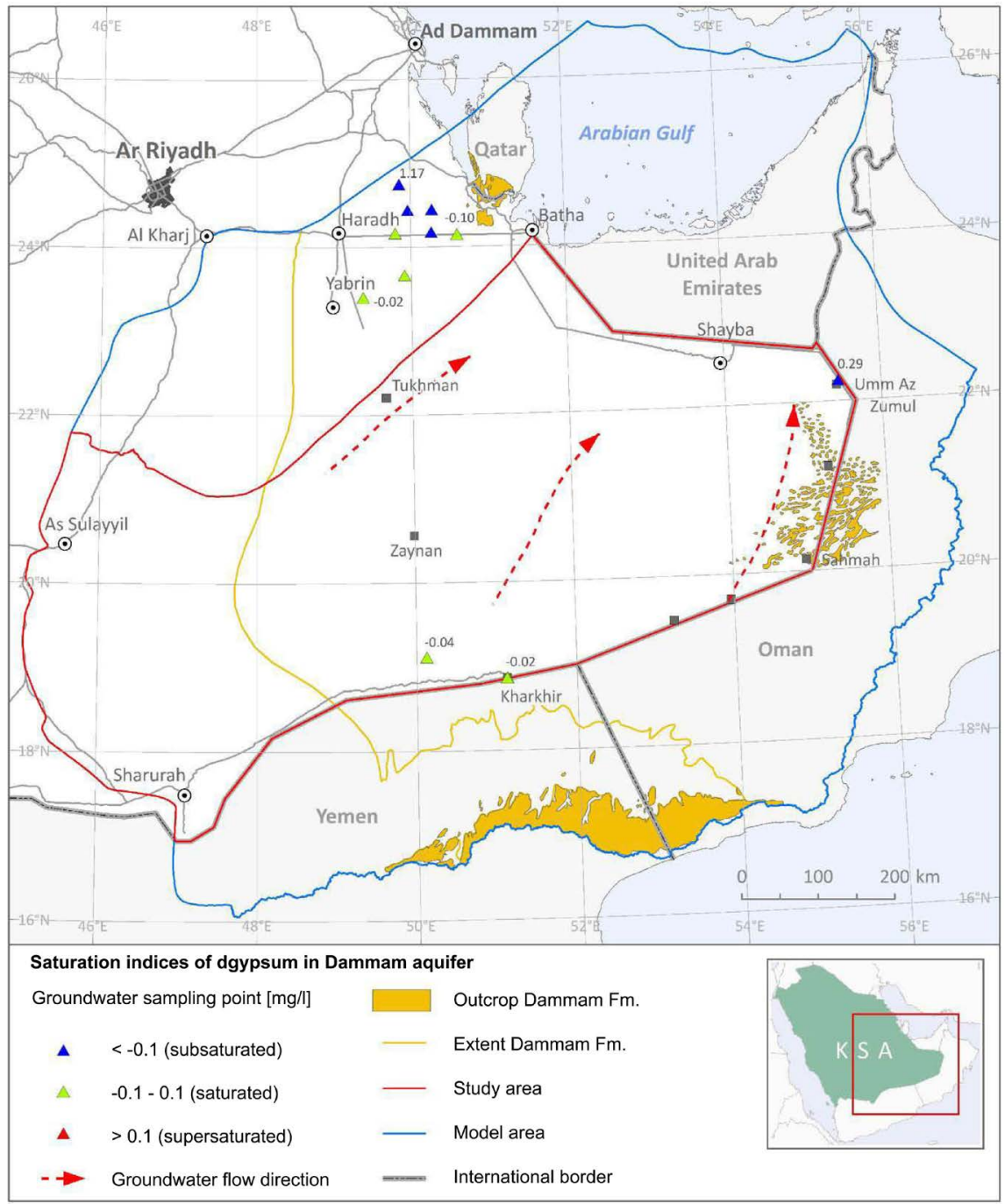

Figure 12. Distribution of the saturation indices of gypsum within Umm Er Radhuma aquifer.

Table 2. Saturation indices for the carbonate minerals.

\begin{tabular}{cccccccc}
\hline Well Name & $\mathrm{O}_{2}(\mathrm{mg} / \mathrm{L})$ & $\mathrm{Fe}^{2+}(\mathrm{mg} / \mathrm{L})$ & SI Calc. & SI Arag. & SI Dolo. SI Anhy. SI Gyp. \\
\hline GHFH-801 & 0.10 & 0.05 & 0.47 & 0.34 & 1.19 & -0.76 & -0.64 \\
OLTD40 & n.d. & 0.10 & 1.39 & 1.27 & 2.88 & -0.81 & -0.75 \\
S-397 & 0.08 & $<0.10$ & 0.42 & 0.28 & 0.88 & -0.24 & -0.09 \\
OLTD13 & 0.13 & 1.03 & 1.58 & 1.44 & 3.19 & -0.44 & -0.27 \\
OLTD52 & 0.67 & 0.03 & 0.35 & 0.21 & 0.74 & -0.30 & -0.15 \\
OLTD39 & 0.06 & 0.03 & 0.48 & 0.35 & 1.01 & -0.78 & -0.75 \\
JZTD2 & 0.58 & 0.02 & -0.04 & -0.18 & 0.10 & -0.40 & -0.22 \\
MKSR-801 & 1.02 & 0.50 & 0.05 & -0.08 & 0.13 & -0.56 & -0.47 \\
7-S-11 & 2.13 & 0.01 & -0.16 & -0.29 & -0.30 & -1.06 & -0.91 \\
7-S-25 & 0.30 & 0.11 & 1.79 & 1.65 & 3.67 & -0.43 & -0.31 \\
SUHL-801 & n.d. & 0.06 & 0.26 & 0.13 & 0.68 & -0.74 & -0.69 \\
\hline
\end{tabular}




\section{Continued}

\begin{tabular}{|c|c|c|c|c|c|c|c|}
\hline Kharkher New Well & 4.96 & 0.18 & 0.22 & 0.09 & 0.62 & -0.76 & -0.65 \\
\hline JZTD1 & 3.67 & $<0.10$ & -0.67 & -0.81 & -1.18 & -1.32 & -1.18 \\
\hline KIDNSRAK-804 & 0.08 & $<0.10$ & 0.80 & 0.68 & 1.65 & -0.36 & -0.27 \\
\hline HFER-RBKL-801 & 6.22 & 0.01 & -0.96 & -1.10 & -1.78 & -1.45 & -1.25 \\
\hline Well Al Bragh & 2.37 & 0.01 & 0.02 & -0.12 & 0.21 & -1.28 & -1.13 \\
\hline HDDH-801 & 0.10 & 0.03 & 0.16 & 0.03 & 0.37 & -0.58 & -0.48 \\
\hline$S-610$ & 0.09 & $<0.10$ & 0.34 & 0.21 & 0.81 & -0.51 & -0.3 \\
\hline S-646 & 0.09 & $<0.10$ & 0.32 & 0.19 & 0.81 & -0.61 & -0.49 \\
\hline S-612 & 0.08 & $<0.10$ & 0.32 & 0.19 & 0.83 & -0.66 & -0.53 \\
\hline Grada-01 & 0.09 & 0.01 & 0.35 & 0.22 & 0.89 & -0.78 & -0.64 \\
\hline Grada-WW1 & 0.09 & $<0.10$ & 0.45 & 0.32 & 1.09 & -0.68 & -0.56 \\
\hline $7-S-64$ & 4.02 & 0.13 & -0.28 & -0.42 & -0.54 & -1.24 & -1.07 \\
\hline Sul_12 & 0.71 & $<0.10$ & 0.07 & -0.07 & -0.02 & -0.58 & -0.38 \\
\hline $7-S-29$ & 0.59 & $<0.10$ & 0.21 & 0.08 & 0.70 & -0.85 & -0.73 \\
\hline W7-S-84 & 0.07 & 0.05 & 0.10 & -0.03 & 0.54 & -1.01 & -0.89 \\
\hline RAK-05E & 1.24 & 0.29 & 0.24 & 0.11 & 0.47 & -0.59 & -0.47 \\
\hline FRIS-801 & n.d. & 0.08 & 0.00 & -0.13 & 0.11 & -0.67 & -0.61 \\
\hline 7-S-XX & 4.42 & 0.02 & 0.08 & -0.05 & 0.33 & -0.73 & -0.63 \\
\hline Kharkher BG Center & 0.08 & 0.45 & 0.03 & -0.10 & 0.22 & -0.65 & -0.55 \\
\hline Kharkher_Desal & 0.46 & 0.44 & -0.40 & -0.53 & -0.65 & -0.65 & -0.55 \\
\hline $7-S-28$ & 0.08 & 0.07 & 0.87 & 0.73 & 2.13 & -1.14 & -0.98 \\
\hline ST16 & n.d. & 0.16 & 0.08 & -0.04 & 0.34 & -0.99 & -0.94 \\
\hline Uum Al Mulah Well & 1.19 & 0.12 & -0.14 & -0.27 & -0.23 & -0.56 & -0.42 \\
\hline $7-S-53$ & 0.78 & 0.02 & -0.52 & -0.65 & -0.86 & -0.80 & -0.72 \\
\hline S-699A & 0.16 & 0.01 & 0.24 & 0.11 & 0.56 & -0.75 & -0.65 \\
\hline S-699A & 4010 & 0.16 & 0.01 & 0.24 & 0.11 & 0.56 & -0.75 \\
\hline S-688A & 0.51 & 0.07 & 0.06 & -0.07 & 0.45 & -1.18 & -1.09 \\
\hline KRKR-803 & 2.87 & 0.07 & 0.01 & -0.12 & 0.35 & -1.05 & -0.95 \\
\hline SRFH-801 & 0.19 & 0.01 & 0.32 & 0.19 & 0.91 & -1.04 & -0.97 \\
\hline JZTD7 & 0.10 & 0.08 & -0.06 & -0.19 & 0.03 & -0.67 & -0.57 \\
\hline S-686 & 0.19 & 0.03 & 0.21 & 0.09 & 0.76 & -0.41 & -0.34 \\
\hline RWKB-801 & 0.55 & 0.01 & 0.59 & 0.46 & 1.35 & -0.90 & -0.79 \\
\hline $7-S-27$ & 0.40 & $<0.10$ & 0.48 & 0.35 & 1.17 & -0.80 & -0.67 \\
\hline RAK-07O & 0.27 & 0.09 & 0.67 & 0.54 & 1.42 & -0.68 & -0.55 \\
\hline RAK-07E & n.d. & 0.03 & 0.01 & -0.11 & 0.09 & -0.67 & -0.68 \\
\hline
\end{tabular}

Table 3. Result of the saturation indices for the Well RAK 05-E.

\begin{tabular}{ccccc}
\hline Phase & $\begin{array}{c}\text { Saturation } \\
\text { Index }\end{array}$ & $\log$ IAP & $\log \mathrm{K}$ & $(316 \mathrm{~K}, 1 \mathrm{~atm})$ \\
\hline $\mathrm{Al}(\mathrm{OH})_{3}$ & -2.26 & 7.43 & 9.69 & $\mathrm{Al}(\mathrm{OH})_{3}$ \\
Albite & -1.19 & -18.11 & -16.92 & $\mathrm{NaAlSi}_{3} \mathrm{O}_{8}$ \\
\hline
\end{tabular}




\section{Continued}

\begin{tabular}{|c|c|c|c|c|}
\hline Alunite & -3.81 & -7.31 & -3.50 & $\mathrm{KAl}_{3}\left(\mathrm{SO}_{4}\right)_{2}(\mathrm{OH})_{6}$ \\
\hline Anhydrite & -0.58 & -5.07 & -4.49 & $\mathrm{CaSO}_{4}$ \\
\hline Anorthite & -2.59 & -21.82 & -19.23 & $\mathrm{CaAl}_{2} \mathrm{Si}_{2} \mathrm{O}_{8}$ \\
\hline Aragonite & 0.20 & -8.27 & -8.47 & $\mathrm{CaCO}_{3}$ \\
\hline Ca-Montmorillonite & 1.67 & -40.92 & -42.59 & $\mathrm{Ca}_{0.165} \mathrm{Al}_{2.33} \mathrm{Si}_{3.67} \mathrm{O}_{10}(\mathrm{OH})_{2}$ \\
\hline Calcite & 0.33 & -8.27 & -8.60 & $\mathrm{CaCO}_{3}$ \\
\hline Chalcedony & 0.25 & -3.10 & -3.35 & $\mathrm{SiO}_{2}$ \\
\hline Chlorite & -1.29 & 60.77 & 62.06 & $\mathrm{Mg}_{5} \mathrm{Al}_{2} \mathrm{Si}_{3} \mathrm{O}_{10}(\mathrm{OH})_{8}$ \\
\hline Chrysotile & -3.17 & 26.92 & 30.09 & $\mathrm{Mg}_{3} \mathrm{Si}_{2} \mathrm{O}_{5}(\mathrm{OH})_{4}$ \\
\hline $\mathrm{CO}_{2}$ (gas) & -1.47 & -3.12 & -1.65 & $\mathrm{CO}_{2}$ \\
\hline Dolomite & 0.64 & -16.84 & -17.48 & $\mathrm{CaMg}\left(\mathrm{CO}_{3}\right)_{2}$ \\
\hline $\mathrm{Fe}(\mathrm{OH})_{3}$ (aqueous) & 1.02 & 5.91 & 4.89 & $\mathrm{Fe}(\mathrm{OH})_{3}$ \\
\hline $\mathrm{FeS}$ & -0.53 & -4.44 & -3.92 & $\mathrm{FeS}$ \\
\hline Gibbsite & 0.27 & 7.43 & 7.16 & $\mathrm{Al}(\mathrm{OH})_{3}$ \\
\hline Goethite & 7.51 & 5.91 & -1.60 & $\mathrm{FeOOH}$ \\
\hline Gypsum & -0.47 & -5.07 & -4.60 & $\mathrm{CaSO}_{4}: 2 \mathrm{H}_{2} \mathrm{O}$ \\
\hline $\mathrm{H}_{2}$ (gas) & -21.99 & -25.12 & -3.13 & $\mathrm{H}_{2}$ \\
\hline $\mathrm{H}_{2} \mathrm{O}$ & -1.07 & -0.00 & 1.07 & $\mathrm{H}_{2} \mathrm{O}$ \\
\hline $\mathrm{H}_{2} \mathrm{~S}$ & -4.06 & -12.02 & -7.96 & $\mathrm{H}_{2} \mathrm{~S}$ \\
\hline Halite & -6.11 & -4.53 & 1.58 & $\mathrm{NaCl}$ \\
\hline Hematite & 17.12 & 11.82 & -5.30 & $\mathrm{Fe}_{2} \mathrm{O}_{3}$ \\
\hline Illite & 0.82 & -37.17 & -37.98 & $\mathrm{~K}_{0.6} \mathrm{Mg}_{0.25} \mathrm{Al}_{2.3} \mathrm{Si}_{3.5} \mathrm{O}_{10}(\mathrm{OH})_{2}$ \\
\hline Jarosite-K & -1.36 & -11.87 & -10.52 & $\mathrm{KFe}_{3}\left(\mathrm{SO}_{4}\right)_{2}(\mathrm{OH})_{6}$ \\
\hline K-feldspar & -0.28 & -19.57 & -19.29 & $\mathrm{KAlSi}_{3} \mathrm{O}_{8}$ \\
\hline K-mica & 5.98 & 16.21 & 10.23 & $\mathrm{KAl}_{3} \mathrm{Si}_{3} \mathrm{O}_{10}(\mathrm{OH})_{2}$ \\
\hline Kaolinite & 2.70 & 8.66 & 5.96 & $\mathrm{Al}_{2} \mathrm{Si}_{2} \mathrm{O}_{5}(\mathrm{OH})_{4}$ \\
\hline Mackinawite & 0.20 & -4.44 & -4.65 & $\mathrm{FeS}$ \\
\hline Melanterite & -6.82 & -8.84 & -2.02 & $\mathrm{FeSO}_{4}: 7 \mathrm{H}_{2} \mathrm{O}$ \\
\hline $\mathrm{O}_{2}$ (gas) & -33.66 & -36.66 & -3.00 & $\mathrm{O}_{2}$ \\
\hline Pyrite & 23.44 & 5.43 & -18.01 & $\mathrm{FeS}_{2}$ \\
\hline Quartz & 0.63 & -3.10 & -3.73 & $\mathrm{SiO}_{2}$ \\
\hline Sepiolite & -2.54 & 12.77 & 15.31 & $\mathrm{Mg}_{2} \mathrm{Si}_{3} \mathrm{O}_{7.5} \mathrm{OH}: 3 \mathrm{H}_{2} \mathrm{O}$ \\
\hline Sepiolite & -5.89 & 12.77 & 18.66 & $\mathrm{Mg}_{2} \mathrm{Si}_{3} \mathrm{O}_{7.5} \mathrm{OH}: 3 \mathrm{H}_{2} \mathrm{O}$ \\
\hline Siderite & -1.05 & -12.04 & -10.99 & $\mathrm{FeCO}_{3}$ \\
\hline $\mathrm{SiO}_{2}$ & -0.53 & -3.10 & -2.57 & $\mathrm{SiO}_{2}$ \\
\hline Sulfur & 12.14 & 16.62 & 4.49 & S \\
\hline Sylvite & -6.97 & -5.98 & 0.98 & $\mathrm{KCl}$ \\
\hline Talc & 1.25 & 20.71 & 19.46 & $\mathrm{Mg}_{3} \mathrm{Si}_{4} \mathrm{O}_{10}(\mathrm{OH})_{2}$ \\
\hline
\end{tabular}




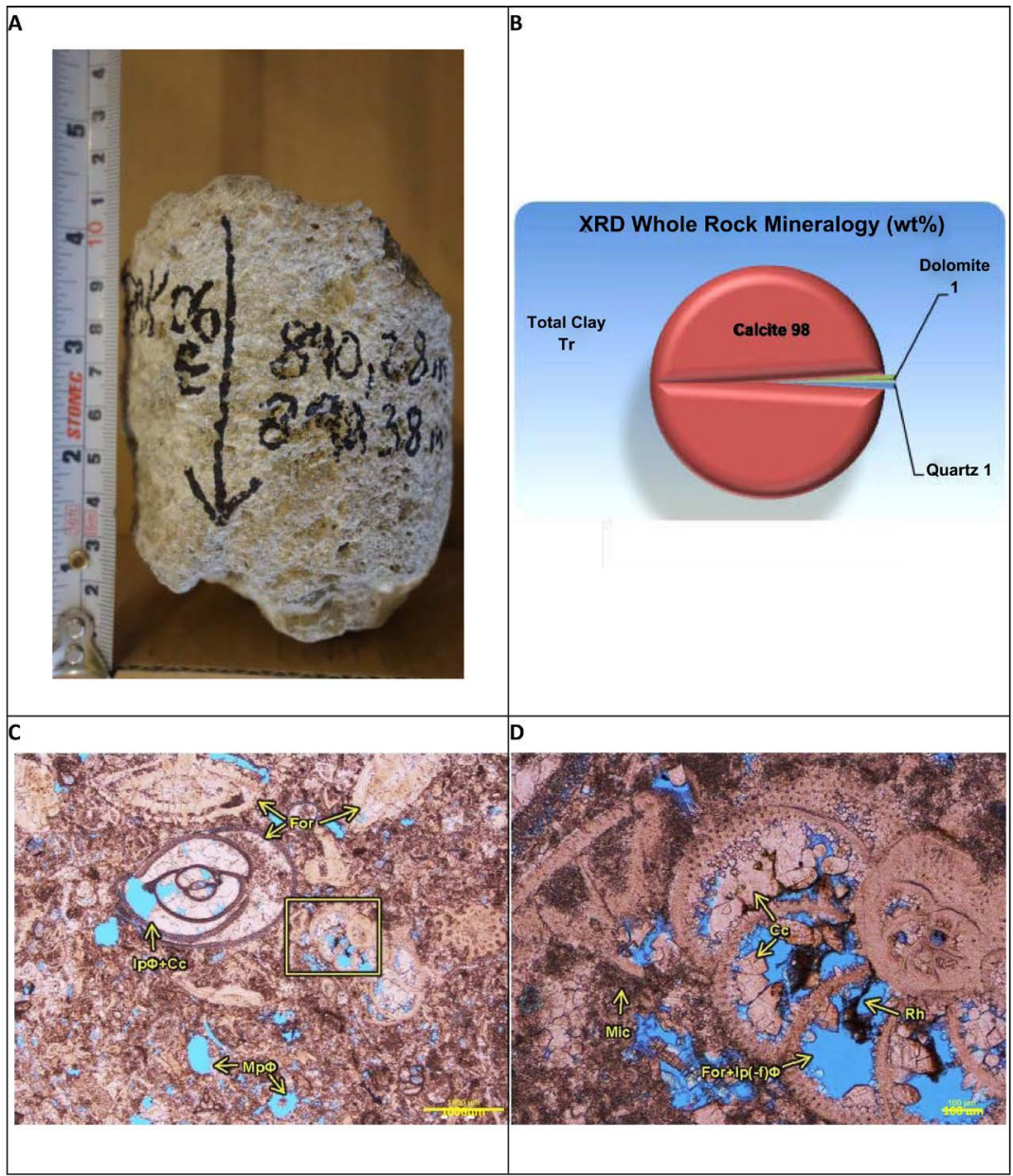

Figure 13. (A) Core sample RAK 06-E. (B) Pie chart showing the results from the X-Ray Diffraction (XRD) Analysis. C: Low magnification view of the sample showing abundant benthic forams (For). A miliolid foram with intraparticle (-fossil) pores partially healed by sparry calcite cement $(\mathrm{Ip}(-\mathrm{f}) \Phi+\mathrm{Cc})$. Minor scattered moldic porosity $(\mathrm{Mp} \Phi)$ observed. (D) High magnification view highlighting a multi-chambered planspiral benthic foram with intraparticle (-fossil) porosity [For+Ip(-f)Ф]. Pore occluding calcite cement (Cc) and rare residual hydrocarbon (Rh) observed. Minor micrite (Mic) matrix noted (MEWA, 2017).

which have major effect on the cation exchange process taking place in the aquifer.

In order to investigate the influence of the cation exchange taking place on the clay minerals in the aquifer, the ratio of $\left[\left(\mathrm{Ca}^{2+}+\mathrm{Mg}^{2+}\right)-\left(\mathrm{SO}_{4}^{2-}+\mathrm{HCO}_{3}^{-}\right)\right]$ against $\left(\mathrm{Na}^{+}-\mathrm{Cl}^{-}\right)$has been applied. Based on Figure 14, most of groundwater samples plot close to 1:1 line, indicating that cation exchange is affecting the hydrochemical composition of the groundwater. Cation exchange can also be quantified by the Chloro-Alkaline indices (CAI) suggested by Schoeller (1977), to indicate ion exchange between the groundwater and its host environment during residence or travel. The value of these indices can be positive or negative. If the value is positive then it explains that the exchange of $\mathrm{Na}^{+}$and $\mathrm{K}^{+}$ions are from water with $\mathrm{Mg}^{2+}$ and $\mathrm{Ca}^{2+}$ ions of the rocks. This indicates a direct base 


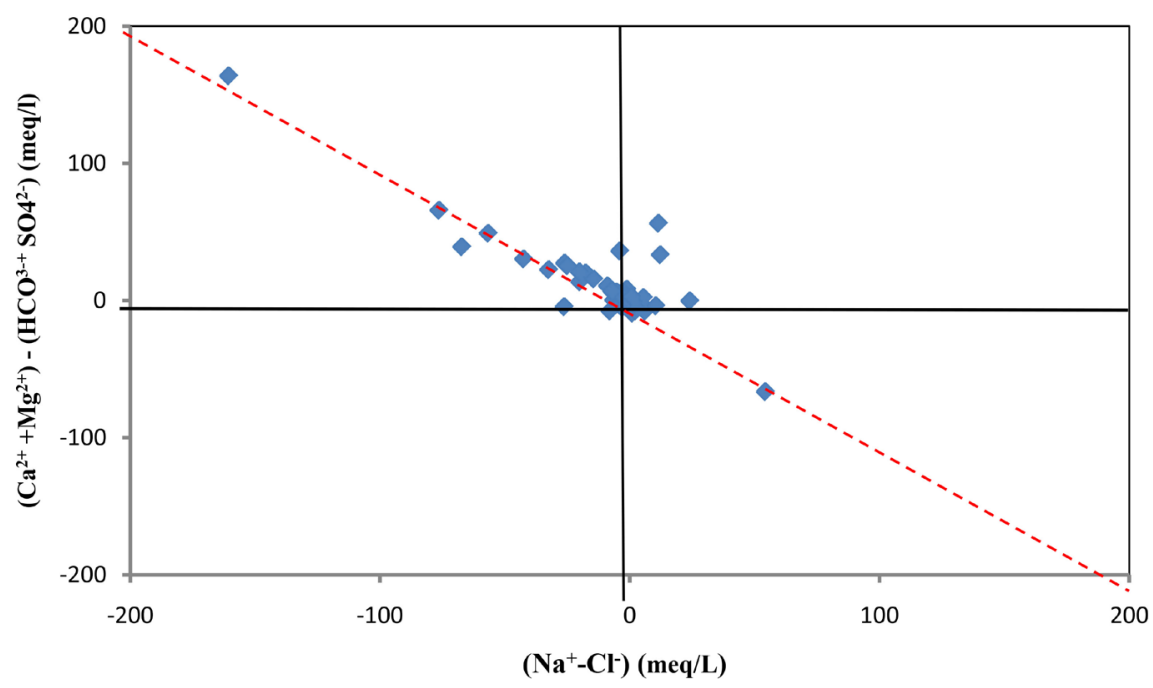

Figure 14. $\left[\left(\mathrm{Ca}^{2+}+\mathrm{Mg}^{2+}\right)-\left(\mathrm{SO}_{4}^{2-}+\mathrm{HCO}_{3}^{-}\right)\right]$against $\left(\mathrm{Na}^{+}-\mathrm{Cl}^{-}\right)$, showing that the effect of cation exchange is active on the hydrochemistry of the groundwater.

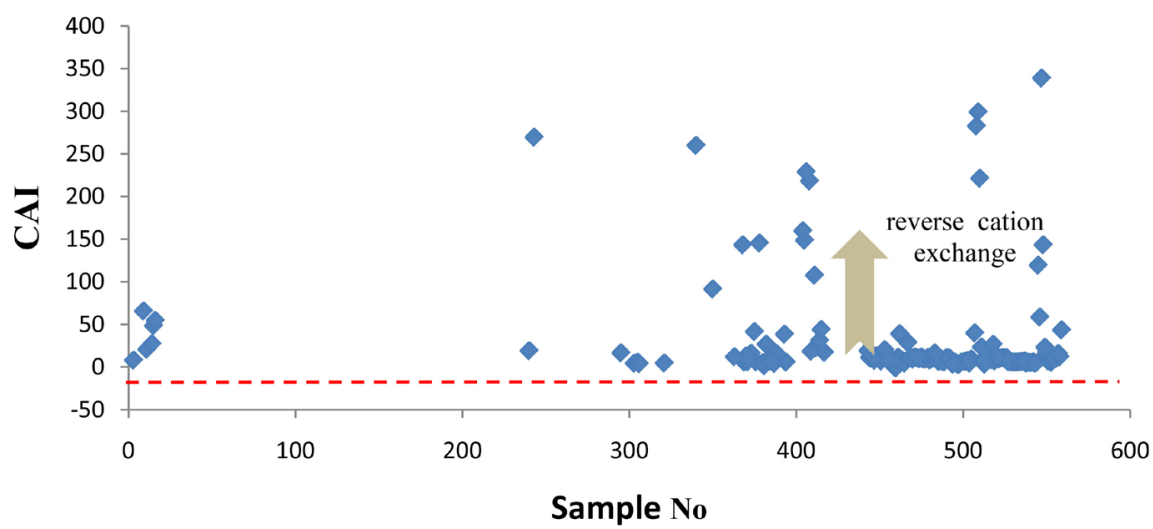

Figure 15. (CAI) or $\left[\mathrm{Cl}^{-}-\left(\mathrm{Na}^{+}+\mathrm{K}^{+}\right)\right] / \mathrm{Cl}^{-}$(in meq/L), the positive values indicate reverse cation exchange, while the negative values indicate cation exchange.

(cation-anion) exchange reaction. In contrast, if the value is negative then it means that there is an exchange $\mathrm{Mg}^{2+}$ and $\mathrm{Ca}^{2+}$ of the water with $\mathrm{Na}^{+}$and $\mathrm{K}^{+}$ions from rocks, so the exchange is in indirect base indicating chloro-alkaline disequilibrium. These reactions are known as cation-anion exchange reaction. The Chloro-Alkaline index (CAI) is defined by the ratio of $\left[\mathrm{Cl}^{-}-\left(\mathrm{Na}^{+}+\mathrm{K}^{+}\right)\right] / \mathrm{Cl}^{-}$(in $\mathrm{meq} / \mathrm{L}$ ). Most of groundwater samples have values greater than zero which indicate reverse cation exchange, where $\mathrm{Ca}^{2+}$ and $\mathrm{Mg}^{2+}$ are being released from the aquifer matrix to groundwater (Figure 15).

The most plausible source of $\mathrm{Na}^{+}$and $\mathrm{Cl}^{-}$is halite dissolution which releases equal molar amounts of $\mathrm{Na}^{+}$and $\mathrm{Cl}^{-}$into groundwater (Equation (6)) Accordingly, samples should plot on the 1:1 line of halite dissolution on the $\mathrm{Na}^{+}$against $\mathrm{Cl}^{-}$plot. According to Figure 16, most of the groundwater samples plot along the 1:1 halite dissolution whilst a few samples plot below the line, which is attributed mostly to reverse cation exchange. 


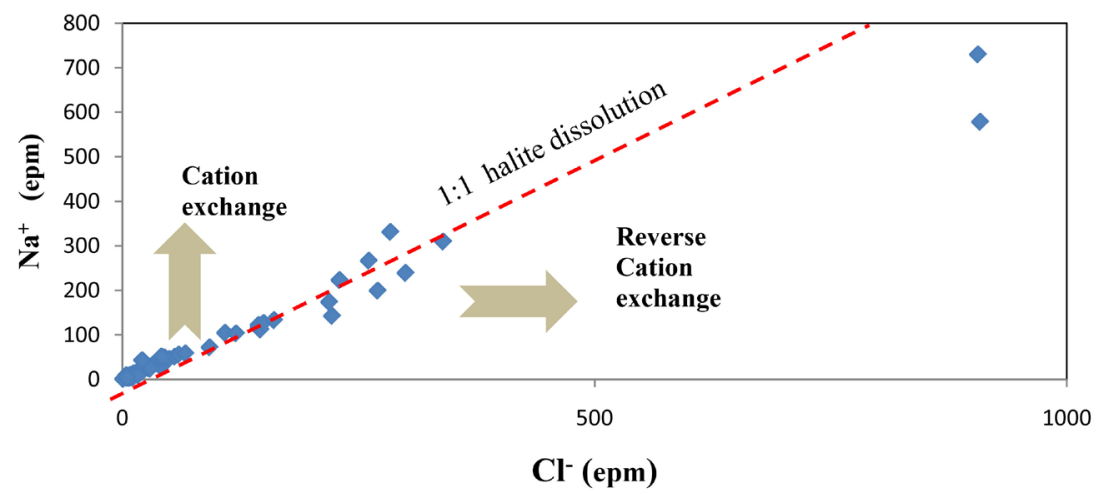

Figure 16. $\mathrm{Na}^{+}$against $\mathrm{Cl}^{-}$relationship, indicating that most of the samples plot on the 1:1 line of halite dissolution which account for $\mathrm{Na}^{+}$and $\mathrm{Cl}^{-}$in groundwater.

$$
\mathrm{NaCl} \rightarrow \mathrm{Na}^{+}+\mathrm{Cl}^{-}
$$

\section{Conclusion}

Groundwater salinity increased from west to east, reaching a TDS of more than 27,000 $\mathrm{mg} / \mathrm{L}$ near the border with the United Arab Emirates with a dominating $\mathrm{Na}^{+}-\mathrm{Ca}^{2+} \mathrm{Cl}^{-}-\mathrm{SO}_{4}^{2-}$ water type. The dissolution/precipitation of carbonates andevaporation and precipitation of minerals, especially anhydrite, gypsum, and halite account mainly for the solutes and salinity in groundwater. In addition, other sources of $\mathrm{Ca}^{2+}$ and $\mathrm{Mg}^{2+}$ in the groundwater along with dolomite and calcium carbonate minerals. Whilst the dissolution of anhydrite contributed more $\mathrm{Ca}^{2+}$ to groundwater. On the other hand, the main clay minerals are kaolinite and gibbsite which affected the cation exchange process as illustrated by the Chloro-Alkaline index (CAI), where most of the groundwater samples had positive values, indicating the effect of ion exchange on groundwater chemistry. In the eastern part of the aquifer, the $\mathrm{Na}^{+}-\mathrm{Ca}^{2+} \mathrm{Cl}^{-}-\mathrm{SO}_{4}^{2-}$ water type dominated as the anaerobic conditions prevailed and the reduction of sulphate took place.

\section{Acknowledgements}

The author would like to express his deepest gratitude to Prof. Martin Keller, for the technical support and guidance to complete this research.

\section{Conflicts of Interest}

The author declares no conflicts of interest regarding the publication of this paper.

\section{References}

Al Bassam, A. M. (1983). A Quantitative Study of Harad Wellfield, Umm-Er-Radhuma Aquifer. M.Sc. Thesis, Athens, OH: Ohio University.

Al Bassam, A. M. (1987). Hydrochemical Computer Modelling in Groundwater Related Problems. Ph.D. Thesis, Birmingham: University of Birmingham.

BRGM (1976). Hydrogeological Investigations of the Al Wasia Aquifer in the Eastern Province of Saudi Arabia. Unpub. Report, Kingdom of Saudi Arabia: Ministry of Agri- 
culture \& Water.

BRGM (1979). New Data on Groundwater Resources of Al Kharj Area. Unpub. Report, Kingdom of Saudi Arabia: Ministry of Agriculture \& Water.

Dinçer, T. A. L., Mugrin, A., \& Zimmerman, U. (1974). Study of the Infiltration and Recharge through the Sand Dunes and Arid Zones with Special Reference to the Stable Isotopes and Thermonuclear Tritium. Journal of Hydrology, 23, 79-109.

https://doi.org/10.1016/0022-1694(74)90025-0

Edda, K., Oswald, S., Wang, W. Q., Kolditz, O., Engelhardt, I., Al-Saud, M. I., \& Rausch, R. (2011). Large-Scale Modeling of the Groundwater Resources on the Arabian Platform. International Journal of Water Resources and Arid Environments, 1, 38-47.

Elango, L., \& Kannan, R. (2007). Rock-Water Interaction and Its Control on Chemical Composition of Groundwater. In D. Sarkar, R. Datta, \& R. Hannigan (Eds,), Developments in Environmental Science (Vol. 5, pp. 229-243). Amsterdam: Elsevier. https://doi.org/10.1016/S1474-8177(07)05011-5

GDC (1979). Final Draft of Umm-Er-Radhuma Study. Unpub. Report, Kingdom of Saudi Arabia: Ministry of Agriculture \& Water.

Iltaloconsult (1969). Water and Agricultural Development Studies for Area TV. Unpub. Report, Kingdom of Saudi Arabia: Ministry of Agriculture \& Water.

MEWA, Ministry of Environment Water and Agriculture (2017). Detailed Groundwater Resources Studies in the Rub' Al Khali Desert, Vol. 9, Groundwater Quality-Hydrochemistry, Riyadh (In Association with Giz/Dci-Gesellschaft Für Internationale Zusammenarbeit/Dornier.

MOWE (2008). Preliminary Investigations of the Umm Er Radhuma and Overlying Aquifers in the Rub' Al Khali Desert. Unpub. Report, Kingdom of Saudi Arabia: Ministry of Agriculture \& Water.

Parkhurst, D. (1995). U.S. Geological Survey (143 p). Water-Resources Investigations Report 95-4227.

Powers, R. W., Ramirez, L. F., Redmond, C. D., \& Berg, E. L. (1966). Geology of the Arabia Peninsula. Sedimentary Geology of Saudi Arabia. U.S. Geological Survey, Professional Paper 560D. https://doi.org/10.3133/pp560D

Schoeller, H. (1977). Geochemistry of Groundwater. In Groundwater Studies-An International Guide for Research and Practice (Chap. 15, pp. 1-18). Paris: UNESCO.

Shampine, W. J., Dinçer, T., \& Noory, M. (1979). An Evaluation of Isotope Concentrations in the Groundwater of Saudi Arabia. In Isotope Hydrology, 1978. Vienna, Austria: IAEA.

Sogreah (1968). Water and Agricultural Development Studies, Area V. Final Report, Kingdom of Saudi Arabia: Ministry of Agriculture \& Water.

Watuki, G. M. G. H. (1968). Preliminary Study of Umm-Er-Radhuma Aquifer. Unpub. Report, Kingdom of Saudi Arabia: Ministry of Agriculture \& Water.

Watuki, G. M. G. H. (1971). Final Report of Water and Wells, Faisal Settlement Project, Haradh. Unpub. Report, Kingdom of Saudi Arabia: Ministry of Agriculture \& Water.

Ziegler, M. A. (2001). Late Permian to Holocene Paleofacies Evolution of the Arabian Plate and Its Hydrocarbon Occurrences. GeoArabia, 3, 445-504. 